\title{
Random, top-down, or bottom-up coexistence of parasites: malaria population dynamics in multi-parasitic settings
}

\author{
Luis Fernando Chaves, ${ }^{1,6}$ Akira Kaneko, ${ }^{2,3,4}$ and Mercedes Pascual ${ }^{1,5}$ \\ ${ }^{1}$ Department of Ecology and Evolutionary Biology, The University of Michigan, Ann Arbor, Michigan 48109-1048 USA \\ ${ }^{2}$ Malaria Research, Unit of Infectious Diseases, Department of Medicine, Karolinska Institutet, 17177 Stockholm, Sweden \\ ${ }^{3}$ Department of International Affairs and Tropical Medicine, Tokyo Women's Medical University, Tokyo 162-8666, Japan \\ ${ }^{4}$ Global COE Program, Nagasaki University, 1-12-4 Sakamoto, Nagasaki 852-8523, Japan \\ ${ }^{5}$ The Howard Hughes Medical Institute, Chevy Chase, Maryland 20815-6789 USA
}

\begin{abstract}
Epidemiological models concerned with the control of malaria using interventions such as bed nets and vaccines increasingly incorporate realistic aspects of malaria biology. The increasing complexity of these models limits their ability to abstract ecological processes and to address questions on the regulation of population dynamics using time-series data, particularly in regards to interactions between different pathogens and the regulatory role of innate (bottom-up) and acquired (top-down) immunity. We use a theoretical framework to test hypotheses on the importance of population-level immunity and parasite abundance in regulating the population dynamics of malaria. We use qualitative loop analyses to examine the sign of the interaction between Plasmodium falciparum and P. vivax at the population level, and we discuss implications of this sign for the within-host regulation of parasites. Our analyses of monthly malaria time-series data from the island of Espirito Santo, Vanuatu (1983-1997), show that the dynamics of $P$. falciparum are not sensitive to $P$. vivax, whereas infections by the latter increase in response to those of the former. These results support a differential use of resources inside the hosts, a resource-consumer interaction between hosts and their immune system, and within-host regulation of parasites. Finally, our results emphasize the need to better understand factors regulating malaria dynamics before developing control strategies and call for the use of control strategies directed at the interruption of transmission, such as vector control and the use of bed nets.
\end{abstract}

Key words: bed nets; cross-immunity; loop analysis; malaria time series; parasite interactions; Plasmodium falciparum; Plasmodium vivax; population regulation.

\section{INTRODUCTION}

Malaria, one of the most devastating infectious diseases in humans, is widely distributed across the tropics. Infections can be caused by four different parasite species: Plasmodium vivax, P. ovale, P. malariae, and $P$. falciparum. In most malaria endemic regions, two or more parasite species co-occur (e.g., Cohen 1973), as well as several strains of any given species (e.g., Färnert et al. 1997). This diversity poses a challenge to our understanding of the population dynamics of the disease, and several scenarios have been proposed to understand how the infection by one parasite species or strain determines the fate of an infection by another.

Classical views on the problem considered patterns of malaria infection to be determined by the action of climatic forces (Gill 1928, 1938). The development of ecological theory for competition (e.g., Gause 1934,

Manuscript received 2 June 2008; revised 25 November 2008; accepted 5 December 2008. Corresponding Editor (ad hoc): S. M. Altizer.

${ }^{6}$ Present address: Department of Environmental Studies, Emory University, Atlanta Georgia 30322 USA.

E-mail: 1fchave@emory.edu
Vandermeer 1969), in addition to a growing body of knowledge on the human immune system, led to the proposal of parasite cross-immunity (also known as heterologous immunity) as a force regulating infection by closely related malaria parasites (Cohen 1973). A major emphasis was placed on the specific (a.k.a., adaptive) immune response (Kuby 1997), which recognizes and neutralizes specific pathogens through the response to parasite specific signals or antigens and the selection of $\mathrm{T}$ and $\mathrm{B}$ cells, also generating a memory that allows to quickly target pathogen-motifs following a reinfection. This within-host mechanism implies a topdown regulation in co-infections because it leads to competition for susceptibles in the process of transmission between hosts. The interaction between parasites in this case is negative (see Fig. 1).

The existence of cross-immunity predicts fewer crossinfections than expected by chance. Following Cohen (1973), several authors supported the existence of crossspecific immunity by analyzing cross-sectional studies and finding the number of cross-infections to be less than random (Richie 1988, McKenzie and Bossert 1997, Smith et al. 2001). This idea also underlies more dynamical approaches to the subject of malaria immu- 

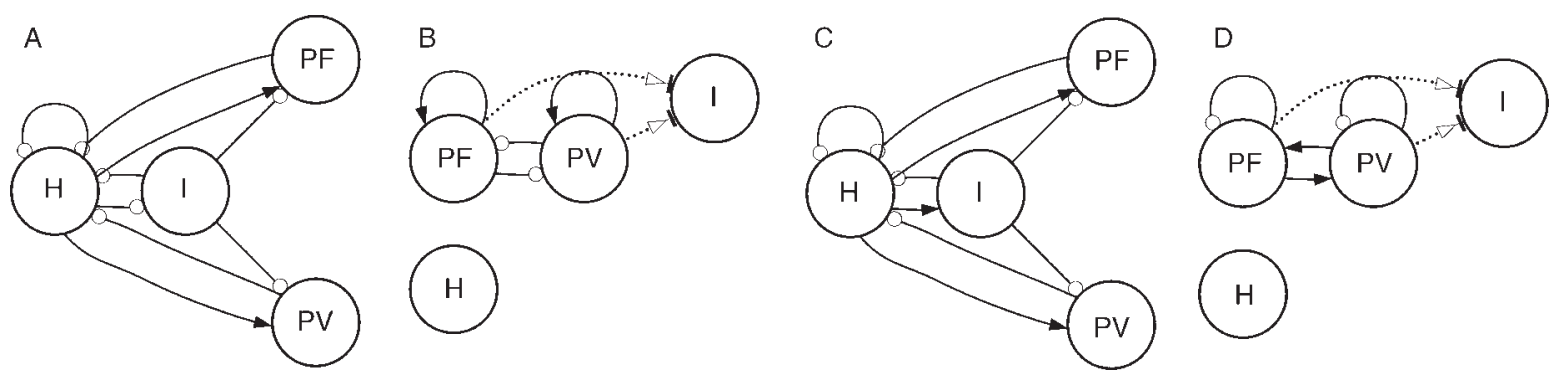

FIG. 1. Feedback loops for top-down and bottom-up regulation of multispecies malaria infections. Negative effects are indicated by small circles, positive effects by arrows, and unknown effects by dotted lines. H represents the hosts, I stands for immunity, and PF and PV represent Plasmodium falciparum and P. vivax, respectively. Immunity is always costly, so under both mechanisms there is a negative effect of I on $\mathrm{H}$, and parasites exploit hosts. (A) Under top-down regulation the interaction of hosts and their immune systems is negative both ways: the interaction of hosts with the parasites determines their immune response, and immunity regulates the hosts available for the parasites at the population level. This corresponds to a mechanism where immunity is regulated by the influx of susceptibles. Therefore, each parasitic infection has a negative effect on the other (B). (C) Under a bottom-up regulation, the hosts feed their immune system when parasitized, since they canalize the information about the infection, resulting in a positive interaction among parasites (D) and the self-regulation of each species. Panels (B) and (D) were obtained through loop analysis (see Puccia and Levins [1985] for details on the technique).

nity and the role of multiple strains of a given parasite species (Gupta and Day 1994a, b). However, as pointed out by Molineaux et al. (1980), Cohen's (1973) method requires high quality data and assumes that all individuals in a population manage infections in the same way. Molineaux et al. (1980) found the number of cross-infections to be higher than expected at random, a pattern that does not rule out cross-immunity, but can arise when some individuals in a population do not raise a proper immune response against the parasites. With the advent of molecular techniques, the pattern described by Molineaux et al. (1980) was shown to be more common than originally suspected, both at the intraspecific (Färnert et al. 1997, Felger et al. 1999, Smith et al. 1999) and interspecific level (Mehlotra et al. 2000, Bruce et al. 2000, Mayxay et al. 2004).

Advances in immunology also showed that immune responses can act through nonspecific or innate mechanisms that do not generate memory cells (Kuby 1997). Before the discovery of nonspecific immunity, dynamic transmission models for malaria developed in the context of the Garki project included hosts whose response to infection differed, closely resembling differences that could arise from innate vs. acquired immunity (Dietz et al. 1974). The Garki project was a large scale intervention in Nigerian villages to test several malaria control measures ranging from vector control with insecticides to drug based malaria chemoprophylaxis. The effectiveness of different control measures was evaluated using a combination of entomological, immunological, parasitological and demographic data (Molineaux and Gramiccia 1980). A mathematical transmission model developed as part of the Garki project assumed some hosts develop temporary immunity to re-infection, as expected under acquired immunity, while others can be readily infected after clearing their parasites, akin to innate immunity
(Dietz et al. 1974). Analysis of the course of individual infections from malariotherapy studies (Molineaux et al. 2002) strongly supported individual heterogeneity in immune responses, with no evidence for cross-specific immunity at the population level.

Nonspecific innate immunity implies a bottom-up regulation mechanism for co-infections, in the sense that hosts can be seen as a self-regulating resource for the parasites (who act as consumers) through the action of the immune system (see Fig. 1). We can also consider that hosts "feed" their immune system when infected at a cost for immunity, which would result in a positive interaction among parasites. The model by Dietz et al. (1974) captured this diversity of mechanisms by subdividing the population according to the handling of infection and the development of immunity across different hosts. This model has been the most successful approximation to malaria dynamics in the populations of the Garki project (Nedelman 1984, 1985, Struchiner et al. 1989). However, epidemiological data on which the model was based are unique in the level of detail. Importantly, the model does not consider the possible effects of environmental forcing on malaria dynamics, whereas recent work has demonstrated that transmission varies with both temperature and rainfall (e.g., Pascual et al. 2008).

As knowledge of intra-host dynamics increase, it is becoming clear that innate immunity can play a key role in the control of malaria infections. Although several models have considered these new findings explicitly (McKenzie and Bossert 2005, Gurarie et al. 2006, Filipe et al. 2007), the question of regulation of parasitic infections has not been addressed using a populationlevel time-series approach. Here, we propose a timeseries modeling approach based on conceptual ideas from population dynamics (Sinclair 1989, Royama 1992, Berryman et al. 2002) that allow us to combine the 
statistical framework of seasonal autoregressive models with mechanistic elements of a simple transmission model. Our model directly incorporates climate forcing and considers the fraction of parasite-free individuals in the population as a limiting resource. This model is used to test three main mechanisms (randomness, top-down or bottom-up regulation) for parasite coexistence in a population of hosts. Specifically, we ask whether models that assume a negative parasite interaction via crossimmunity, a positive parasite interaction via innate immunity, or no immune-mediated interaction provide a better fit to patterns observed for two malaria species, Plasmodium vivax and P. falciparum, in a Pacific Island.

\section{Methods}

\section{Loop analysis for qualitative understanding of species interactions}

Interactions among species can be studied through analysis of a community matrix that describes the interactions of members around an equilibrium (Levins 1968 , 1974). To apply loop analysis, the first step is to define interactions among the system's components. For the multispecies malaria systems described in Fig. 1, the components are PV (Plasmodium vivax), PF ( $P$. falciparum), $\mathrm{H}$ (hosts), and I (immunity). Let $\phi_{i j}$ denote the qualitative effect of $i$ on $j$ represented by a negative or positive sign corresponding respectively to a decrease or an increase in $j$ when $i$ increases. Then, all interactions can be represented in a community matrix as follows:

$$
\boldsymbol{\phi}=\left(\begin{array}{llll}
\phi_{\mathrm{HH}} & \phi_{\mathrm{IH}} & \phi_{\mathrm{PFH}} & \phi_{\mathrm{PVH}} \\
\phi_{\mathrm{HI}} & \phi_{\mathrm{II}} & \phi_{\mathrm{PFI}} & \phi_{\mathrm{PVI}} \\
\phi_{\mathrm{HPF}} & \phi_{\mathrm{IPF}} & \phi_{\mathrm{PFPF}} & \phi_{\mathrm{PVPF}} \\
\phi_{\mathrm{HPV}} & \phi_{\mathrm{IPV}} & \phi_{\mathrm{PFPV}} & \phi_{\mathrm{PVPV}}
\end{array}\right) .
$$

Assuming that hosts self-regulate (e.g., Cohen 1995), $\phi_{\mathrm{HH}}$ is negative. By definition, it also follows that $\phi_{\mathrm{PFH}}$ and $\phi_{\mathrm{PVH}}$ are negative and that $\phi_{\mathrm{HPF}}$ and $\phi_{\mathrm{HPV}}$ are positive, since parasites exploit hosts. Because hosts attack their parasites through immunity, we assume that $\phi_{\text {IPF }}$ and $\phi_{\text {IPV }}$ are negative. We also assume that immunity is costly to hosts and $\phi_{\mathrm{IH}}$ is negative, since resources that can be allocated to grow or reproduction are instead allocated to build up the immune response (e.g., Cannon 1958, Mardsen 1964). At the population level, the effect of host abundance on immunity levels has been typically modeled as negative (e.g., Finkestädt and Grenfell 2000) by assuming that the influx of susceptible hosts regulates population-level immunity; thus $\phi_{\mathrm{HI}}$ is negative. From these considerations (summarized in Fig. 1A), the matrix of signs, $\phi$, becomes

$$
\boldsymbol{\phi}=\left(\begin{array}{cccc}
- & - & - & - \\
- & 0 & 0 & 0 \\
+ & - & 0 & 0 \\
+ & - & 0 & 0
\end{array}\right)
$$

On the other hand, we could assume that immunity is canalized through the host itself when infected (Schmal- hausen 1949, Matzinger 1994). We can then hypothesize that hosts "feed" their immunity leading to a positive value for $\phi_{\mathrm{HI}}$. In this view (summarized in Fig. 1C), $\phi$ is given by

$$
\phi=\left(\begin{array}{cccc}
- & - & - & - \\
+ & 0 & 0 & 0 \\
+ & - & 0 & 0 \\
+ & - & 0 & 0
\end{array}\right)
$$

Once the community matrix is defined, the next step is to compute the community effect matrix, which is the negative inverse of the community matrix, i.e, $-\boldsymbol{\phi}^{-1}$. The community effect matrix shows the direction of change in abundance of the community members following a small perturbation, where indirect effects can also be considered (for formal derivations and extensions see Levins 1974, Puccia and Levins 1985, Zavaleta and Rossignol 2004). The community effect matrix for Eqs. 2 and 3 is summarized in graph form in Fig. 1B, D, which correspond respectively to the analysis of matrices Eqs. 2 and 3.

\section{Model and Theoretical Considerations}

Let $I_{t}$ represent new malaria cases during a discrete time step $\Delta t$ from $t-1$ to $t$, and assume that this quantity changes according to a per capita growth rate $r(t)=\ln \left(I_{t} / I_{t-1}\right)$, which is constant for $\Delta t$ (Turchin 2003). The dynamics for new cases can be written as

$$
I_{t}=I_{t-1} e^{r(t)}
$$

To define $r(t)$, we can equate it to the difference between the rates for recruitment of new infected individuals $(b)$ and recovery of those already infected $(\delta)$, such that $r(t)=b-\delta$. We can also consider a general mass action transmission (de Jong 1995) and let $b=$ $\beta S / N$, (where $\beta$ is the transmission rate, $N$ is the total population size, and $S$ is the susceptible population size). The susceptible class is given by $S=N-I^{\prime}$, where $I^{\prime}$ is defined as the number of parasitemic hosts based on previous and current cases, $I_{t}^{\prime}=f\left(I_{t-i}, i \geq 0\right)$. The parasitemic class $\left(I_{t}^{\prime}\right)$ is introduced to allow for a time of parasite clearance which is longer than the length of the time step $\Delta t$. This class $\left(I_{t}^{\prime}\right)$ is also necessary to account for regulation in the recruitment of new infected individuals by the number of susceptibles. Finally, exogenous forcing, exo $F(t)$, can be added within $r(t)$ to account for effects of extrinsic variables on transmission (Levins 1969, Lewontin and Cohen 1969). Then, the growth rate $r(t)$ is defined as follows:

$$
r(t)=\beta-\delta-\beta \frac{I_{t-1}^{\prime}}{N_{t-1}}+\alpha \operatorname{exo} F_{t}+w_{t}
$$

where $w_{t}$ is a normal random variable accounting for unexplained variation. To simplify the notation in Eq. 5, we define $\beta^{\prime}=\beta-\delta$, as the difference between the transmission rate and the recovery rate. The basic model in discrete time becomes the following: 


$$
I_{t}=I_{t-1}^{\theta} \exp \left(\beta^{\prime}-\beta \frac{I_{t-1}^{\prime}}{N_{t-1}}+\alpha \operatorname{exo} F_{t}+w_{t}\right)
$$

where $0<\theta<1$ is included to consider deviations from mass-action in transmission (Liu et al. 1987). This exponent can also be interpreted as a density-dependent factor in the generation of new infections (Hochberg 1991, Fenton et al. 2002), or as an implicit representation of the effect of spatial and network clustering of the hosts during the process of infection (Finkenstädt and Grenfell 2000, Roy and Pascual 2006). Note that by tracking the growth of new cases, the model explicitly considers the limiting effect of the number of hosts that can be infected (i.e., those that are susceptible).

Seasonality.-Classical work on the dynamics of infectious diseases interprets seasonality as deviations from a mean annual value that occur every year approximately after a fixed period (Gill 1928). Here we modify $r(t)$ to make it a seasonal function:

$$
r(t)=r_{\text {seas }}(k)+\beta^{\prime}-\beta \frac{I_{t-1}^{\prime}}{N_{t-1}}+\alpha \operatorname{exo} F_{t}+w_{t}
$$

where $r_{\text {seas }}(k)$ accounts for the seasonal contribution during the $k$ th period of any given season. Most models incorporate seasonal variation in transmission using fixed seasonalities (e.g., Finkenstädt and Grenfell 2000) or symmetrical sine and cosine functions (e.g., Kot et al. 1988). This approach can lead to symmetrical cyclical behavior that lacks the inherent seasonal variability of a time series, limiting the ability of models to reduce the variability due to unknown factors (Priestley 1988). Seasonal autoregressive forms (Shumway and Stoffer 2000) overcome this limitation by letting the value of a variable $x$ at time $t$ be a function of its previous seasonal value $(t-\sigma)$. Here, $r_{\text {seas }}(k)$ can be defined as a function of $r(t-\sigma)$. When this function can be linearized (using a parameter $\varphi), r_{\text {seas }}(k)$ becomes

$$
r_{\text {seas }}(k)=\varphi \log \left(I_{k-\sigma}\right) .
$$

Then the seasonal version of the model in Eq. 3 can be written as

$$
I_{t}=I_{t-1}^{\theta} I_{t-\sigma}^{\varphi} \exp \left(\beta^{\prime}-\beta \frac{I_{t-1}^{\prime}}{N_{t-1}}+\alpha \operatorname{exo} F_{t}+w_{t}\right) .
$$

Parasite clearance and immunity.-We consider variation among individuals in the duration of parasite clearance (with or without the generation of long lasting immunity) by introducing a random variable $d$ whose distribution describes the length of individual parasitaemia. We let

$$
I_{t}^{\prime}=\sum_{i=0}^{\infty}[1-\operatorname{cmf}(i)] \times I_{t-i}
$$

where $\mathrm{cmf}$ is the cumulative mass function of the random variable $d$ at time $i$, and $I_{t-i}$ is the number of new cases at time $t-i$.

Randomness, top-down, and bottom-up mechanisms.The model presented in Eq. 6 can be extended to account for possible interspecific interactions between two parasites, by introducing a coefficient $\left(\beta_{2}\right)$ for the second species as follows:

$$
I_{t}=I_{t-1}^{\theta} I_{t-\sigma}^{\varphi} \exp \left(\beta^{\prime}-\beta \frac{I_{t-1}^{\prime}}{N_{t-1}} \pm \beta_{2} \frac{I_{2 t-1}^{\prime}}{N_{t-1}}+\alpha \operatorname{exo} F_{t}+w_{t}\right)
$$

where $\beta_{2}$ represents the effect of the second species on the per capita growth of the first one, which can be positive or negative depending on the mechanism of regulation. Thus, the following possibilities correspond to the different mechanisms we wish to test for: (1) if the estimated parameters, $\hat{\beta}=\hat{\beta}_{2}=0$, then random interactions are supported; (2) if $\hat{\beta}<0$; $\hat{\beta}_{2}<0$ (both coefficients with negative signs) cross-specific immunity is the most likely mechanism, since the interaction of the parasites have the signs expected under feedback loops of top-down regulation (i.e., the effect of the second species on the focal is negative, see Fig. 1); (3) if $\hat{\beta}<0$; $\hat{\beta}_{2}>0$, (the focal species with a negative coefficient, the second species with a positive one), nonspecific immunity is at play, since the interactions of the parasites have the signs expected under feedback loops of bottom-up regulation (i.e., the effect of the second species on the first is positive see Fig. 1).

We refer to the dynamical sufficiency (sensu Lewontin 1974) of a given species when there is no significant effect of the other species on its dynamics. In the specific case of malaria, this can reflect niche differences in host resource exploitation since $P$. falciparum is able to colonize erythrocytes (red blood cells) of all ages, whereas $P$. vivax only colonizes young ones, which makes it potentially sensitive to the patterns of exploitation by $P$. falciparum (McQueen and McKenzie 2006).

\section{Data}

Monthly records of malaria in Espirito Santo, Vanuatu were obtained from people attending government health centers (free of charge) who presented with fever or a recent history of fever, and whose standard blood slide analysis indicated infection with either Plasmodium vivax or P. falciparum, from January 1983 to December 1997. Additional data on distributed insecticide treated nets and population growth were available for the same period and obtained from the Malaria and other Vector Borne Diseases Control Unit, Ministry of Health, Port Vila, Vanuatu (Figs. 2 and 3). Seasonal patterns for malaria cases are presented in Fig. 2. Data collection was done under the guidance of the World Health Organization. The Malaria and other Vector Borne Diseases Control Unit maintained quality controls on the reporting system and diagnosis reliability during the studied period. We chose this data set as it is one of the few high quality long term data sets that track infection by both $P$. vivax and $P$. falciparum. 

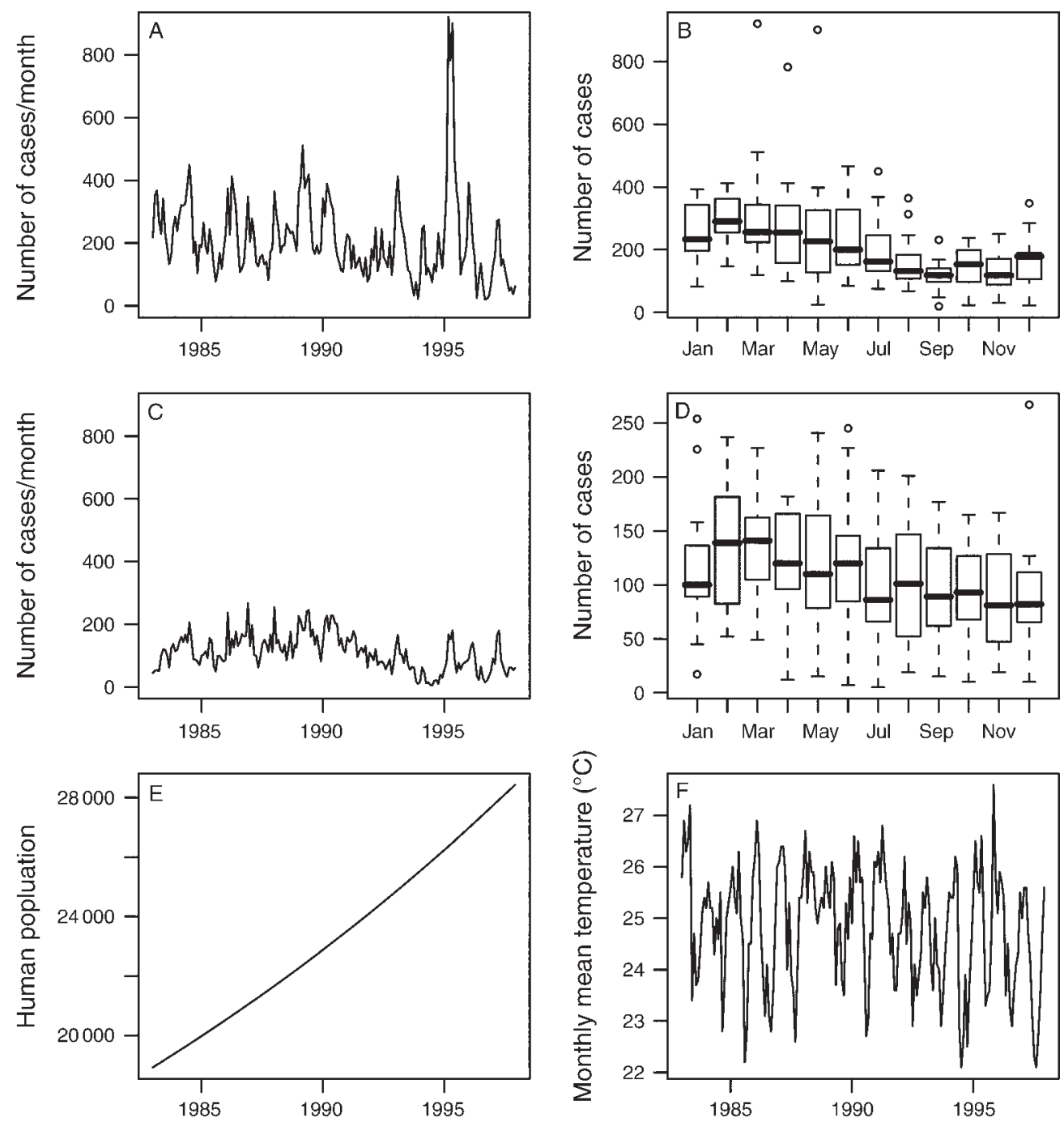

FIG. 2. Time-series patterns of malaria infection, human population, and temperature. For Plasmodium falciparum malaria, (A) time series of monthly incidence in Espirito Santo, Vanuatu (1983-1997) and (B) seasonal pattern of incidence based on monthly averages. For P. vivax malaria, (C) time series of monthly incidence and (D) seasonal pattern of incidence. (E) Population size of Espirito Santo, Vanuatu based on census data, and (F) average monthly temperature from Pekoa Airport. In panels B and D, the solid horizontal bar represents the median values, and the box extends from the first quartile to the third quartile; the whiskers extend to the 5 th and 95 th percentiles, with outliers shown as open dots.

To estimate the distribution of the clearance time $d$ we used the data from $P$. falciparum malariotherapy for neurosyphilis patients published by Collins and Jeffery (1999b), and analyzed by Sama et al. (2006). We considered the datasets from Georgia and South Carolina (Fig. 3C, D), as examples of populations with a minimum use of drugs and some use of drugs, respectively. Since no similar data are available for $P$. vivax we assumed the clearance time distributions to be the same as for P. falciparum. We used this data set because, to the best of our knowledge, it is the only reliable source available to estimate $d$ (Sama et al. 2006). Our use of these data to estimate $d$ relies on the assumption that malaria parasite clearance was inde- pendent of the co-infection with syphilis and that the values of this parameter can be transferred to a geographically and historically unrelated population.

For the climate data, we used records from the airport of Pekoa (Fig. 3D), a close-by island (data available online). ${ }^{7}$ Missing data were imputed from a time series for the political area of Vanuatu (Mitchell et al. 2002). For the analysis, the temperature series was demeaned. Because the model includes exogenous forcing by climatic factors explicitly, the seasonality term of Eq. 8 can account for a series of additional factors that are seasonal but independent of climate, such as harvest

\footnotetext{
${ }^{7}\langle$ http://weather.noaa.gov/ $\rangle$
} 

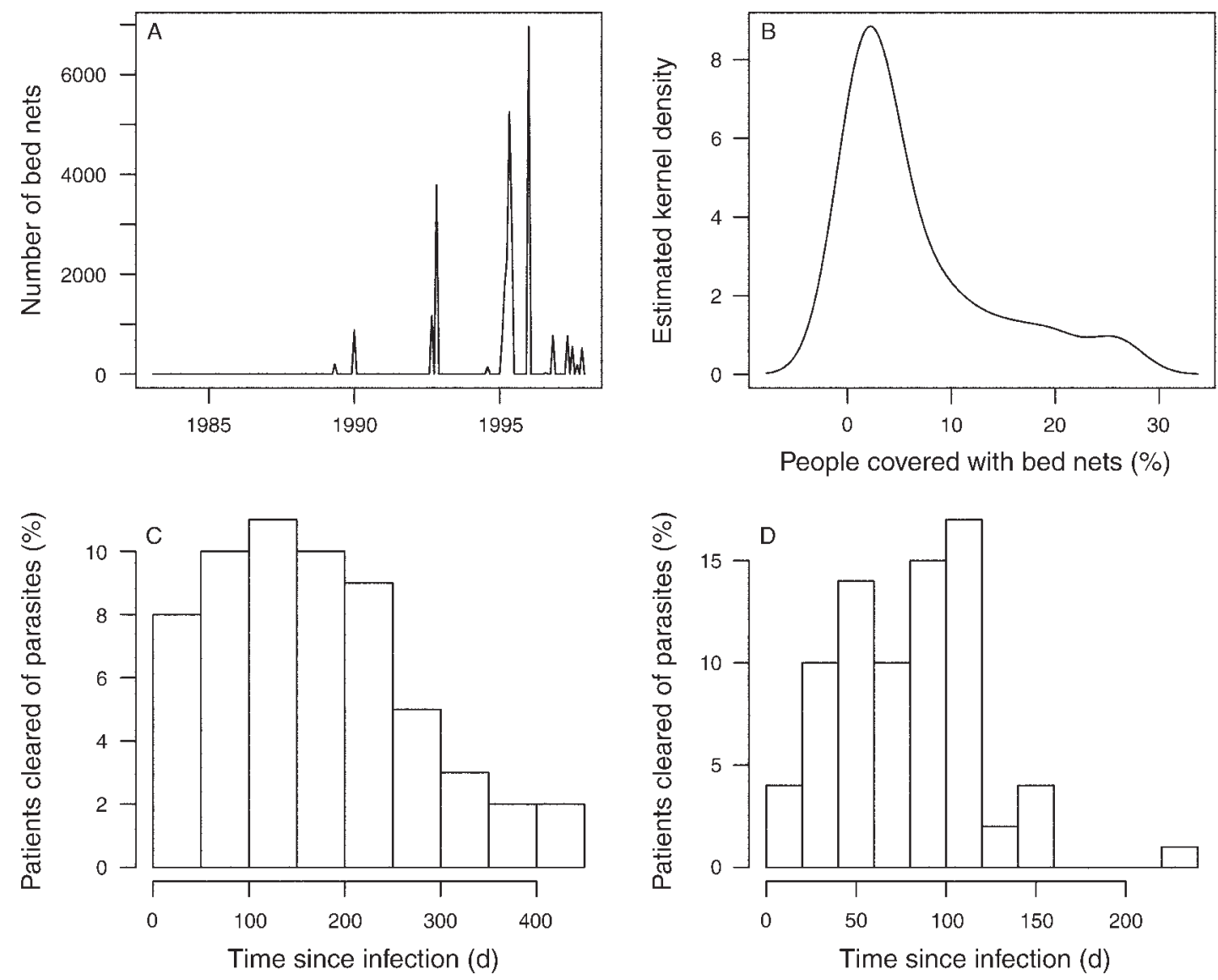

FIG. 3. Bed nets and parasite clearance. (A) Monthly number of bed nets distributed in Espirito Santo, Vanuatu, (B) estimated percentage of people locally covered with each bed net distribution (note that negative values for the percentage of covered people are artifacts of the kernel computation), (C) long-time parasite clearance of $P$. falciparum for Georgia patients without antimalarial therapy, and (D) short-time parasite clearance for South Carolina patients in the presence of anti-malarial therapy.

times, migrations for seasonal work or school terms (Chaves 2007).

\section{Model fitting}

Estimation of $d$ and $I^{\prime}$.- Unlike Sama et al. (2006), who used continuous distributions, we fitted a discrete distribution to clearance time data, given the discrete nature of the data and the way we computed $I^{\prime}$. Negative binomial distributions maximized the likelihood when compared with other discrete distributions (e.g., Poisson). $I^{\prime}$ was obtained with Eq. 10 and the series of $I$ for each species, with month length based on each calendar year, the $\mathrm{cmf}$ was truncated at the end of a calendar year (365 days).

Inapparent infections. -We considered the effect of inapparent infections on the series by multiplying the $I^{\prime}$ series by the ratios of reported to unreported cases published for Espirito Santo, Vanuatu (1992-1994) by Maitland et al. (1996) of $1: 1$ in the wet season (November-May) and 1:4 in the dry season (JuneOctober) for $P$. falciparum, and 1:1 in the wet season and $1: 2$ in the dry season for P. vivax.
Effect of bed nets.- To account for the effects of bed nets we considered two possibilities; bed nets either diminish the size of $N$, the total host population, or they decrease $I^{\prime}$. In both cases the effects were assumed to be additive, i.e., $N_{\mathrm{b}}=N-$ (number of bed nets), $I_{\mathrm{b}}^{\prime}=I^{\prime}-$ (number of bed nets). We assumed the effect of bed nets was cumulative or transient (just for the month when they were delivered). We also computed the percentage of the population covered with insecticide treated bed nets for each delivery (Fig. 3B) and assumed the effects of the bed nets lasted from 1 to 6 months, the life span of the insecticide Permethrin (Kaneko et al. 1998).

Exogenous forcing.-To find appropriate lags for the introduction of climatic variables we used the prewhitening method. This is a filtering technique based on Kalman recursions (Durbin and Koopman 2001), whose outcome is a series of residuals (or innovations) that can be used to perform cross correlation analysis between the series used to estimate the coefficients for the filter and the filtered series, in our case the malaria and temperature time series respectively (see Chaves and Pascual 2006 for an example). More specifically, the 
Kalman recursions provide a prediction-correction method for parameter estimation. For a given time $t$ the mean value and variability of a state vector are estimated for a future time $t+k$. These estimated values are updated at time $t+k$ by using the observation at such time for future predictions. The differences between observation and prediction, i.e., residuals or "innovations," are used to correct the state vector of estimated parameters (Cazelles and Chau 1997). As a result, the cross-correlation function is then computed between the two series but with the intrinsic variability of the filtered series removed, thus allowing the identification of the lags when both series are correlated (Durbin and Koopman 2001, Chaves and Pascual 2006).

Parameter estimation.-For parameter estimation, we linearized the model in Eq. 11 as follows:

$$
\begin{aligned}
\log \left(I_{t}\right)= & \theta \log \left(I_{t-1}\right)+\varphi \log \left(I_{t-12}\right)+\beta^{\prime} \\
& -\beta \frac{I_{t-1}^{\prime}}{N_{t-1}} \pm \beta_{2} \frac{I_{2 t-1}^{\prime}}{N_{t-1}}+\alpha T_{t-5}+w_{t}
\end{aligned}
$$

and used a fitting procedure for negative binomial generalized linear models (NB-GLM) for the time series of each species. To avoid confusion in the parameters, specifically the finding of parameters with incorrect signs or magnitude, a problem arising from the linear algebra behind linear models (Ellner et al. 2002), the estimation of parameter $\beta$ was restricted between $(-\infty,-1]$, using the Nelder-Mead algorithm (Nelder and Mead 1965). The reason for the restriction is that the parameter $\beta$ needs to be larger than the recovery rate, given the endemicity of the disease. Since the recovery rates are equal or smaller than one (the average duration of infections is larger than 3 months and therefore longer than the time step of 1 month), then the estimated value of this parameter, $\hat{\beta}$, must be smaller than -1 . Also, a positive value of $\hat{\beta}$ lacks a plausible biological interpretation since this coefficient represents the magnitude of the negative feedback regulating infections in Eq. 12. The significance of $\beta$ was tested through a maximum likelihood ratio test between models including/excluding the parameter value found with the Nelder-Mead algorithm. However, we could not compute its standard error since the value is fixed as an offset during the final estimation of all other parameters. Since likelihood ratio tests for NB-GLMs are only reliable for a fixed overdispersion parameter $(k)$, we compared the most complex model with simplified ones in which the overdispersion parameter was fixed (at the value of the most complex one). To ensure robustness, we also made comparisons the other way around. The models were fitted only to the data from January 1985 to December 1997, given the need for burning values in $I$ to compute $I^{\prime}$ using Eq. 10.

\section{RESULtS}

The three main mechanisms of within-host interactions between malaria parasites predict very specific outcomes. Under the random hypothesis, only exogenous forcing will be important for the dynamics. Bottom-up regulation implies a positive value for the parameter $\beta_{2}$, reflecting the positive effect of the second parasite on the focal one. For the top-down mechanism, $\beta_{2}$ is expected to be negative. Finally, there is the possibility of dynamical sufficiency for the case of bottom-up regulation, when the dynamics reflect differences in resource exploitation by parasites. This can be tested using the estimated value of the parameter $\beta_{2}$ which would not be different from zero for any of the two parasite species. Fig. 1 shows the loop analysis results for the sign of the interaction between parasites, conditioned on the sign of the relationship between hosts and immunity.

To specify the time-series models, we need both the number of bed nets over time and the independent estimates of clearance times. Fig. 3A shows that the deployment of bed nets was extremely episodic, with bed net distribution following an erratic pattern. Fig. 3B shows the proportion of people locally covered with bed nets, which was on average about $5 \%$ of the island population. Fig. 3C, D shows the distribution of clearance times in neurosyphilis patients treated with malariotherapy. For Georgia patients, long-time clearance time was 169.15 days (close to 6 months), with an overdispersion parameter of 2.10. For South Carolina patients, short-time clearance time was 78.40 days $(\sim 3$ months) with an over-dispersion parameter of 3.51 .

Table 1 shows the search for the best model under the scenarios considered and provides evidence of the robustness of the findings. For both species, consideration of inapparent infections increases the likelihood (minimizes the AIC) of the models. A similar result was found for the long-time clearance, which is partially shown for the best models in Table 2. For P. falciparum, all models without $P$. vivax outperformed the corresponding models with both parasite species. For $P$. vivax the opposite result was found, with models that consider the parasitemic individuals for $P$. falciparum exhibiting the highest likelihood. For both species, the likelihood was also maximized when the effect of bed nets was considered transient and short, of only one month (Table 1). In all cases the restricted search for $\beta$ ended in -1 , and this value was statistically significant as revealed by the likelihood ratio test $(P<0.05)$. Only for $P$. vivax $\beta_{2}$ was statistically significant and positive in the final model (Table 2).

Temperature was a statistically significant driver for the dynamics of both species with a lag of 5 months. For both species the magnitude of this forcing is small, about $10 \%$, when compared with the parameter $\beta$, the difference between transmission and recovery (Table 2). In absolute terms the effect of temperature is slightly larger, about $25 \%$, for $P$. falciparum $(\hat{\alpha} \cong 0.11)$, than for P. $\operatorname{vivax}(\hat{\alpha} \cong 0.08)$.

Finally, Fig. 4 shows the success of the best models in fitting the data. The correlation between observed and 
TABLE 1. Model search outline for two species of malaria parasite, Plasmodium falciparum and $P$. vivax.

\begin{tabular}{|c|c|c|c|}
\hline Species $1 \times$ species 2 & $\begin{array}{l}\text { Inapparent } \\
\text { infections }\end{array}$ & Bed nets & AIC \\
\hline Plasmodium falciparum $\times P$. vivax & yes & $\begin{array}{l}N_{\mathrm{b}} \text { (constant } 1 \text { month) } \\
N_{\mathrm{b}} \text { (cumulative) } \\
I_{\mathrm{b}} \text { (constant } 1 \text { month) } \\
I_{\mathrm{b}} \text { (cumulative) } \\
\text { no effect } \\
N_{\mathrm{b}} \text { (constant } 1 \text { month) } \\
N_{\mathrm{b}} \text { (cumulative) } \\
I_{\mathrm{b}} \text { (constant } 1 \text { month) } \\
I_{\mathrm{b}} \text { (cumulative) } \\
\text { no effect }\end{array}$ & $\begin{array}{l}1916.3 \\
\text { NC } \\
1770.1 \\
1838.9 \\
1772.9 \\
1769.9 \\
1763.4 \\
1768.7 \\
1813.0 \\
1771.7\end{array}$ \\
\hline Plasmodium falciparum $\times$ none & yes & $\begin{array}{l}N_{\mathrm{b}} \text { (constant } 1 \text { month) } \\
N_{\mathrm{b}} \text { (cumulative) } \\
I_{\mathrm{b}} \text { (constant } 1 \text { month) } \\
I_{\mathrm{b}} \text { (cumulative) } \\
\text { no effect } \\
N_{\mathrm{b}} \text { (constant } 1 \text { month) } \\
N_{\mathrm{b}} \text { (cumulative) } \\
I_{\mathrm{b}} \text { (constant } 1 \text { month) } \\
I_{\mathrm{b}} \text { (cumulative) } \\
\text { no effect }\end{array}$ & $\begin{array}{l}1908.1 \\
\text { NC } \\
1772.0 \\
1814.6 \\
1766.4 \\
\mathbf{1 7 6 2 . 9} \\
1765.4 \\
1770.7 \\
1813.0 \\
1765.2\end{array}$ \\
\hline Plasmodium vivax $\times P$. falciparum & yes & $\begin{array}{l}N_{\mathrm{b}} \text { (constant } 1 \text { month) } \\
N_{\mathrm{b}} \text { (cumulative) } \\
I_{\mathrm{b}} \text { (constant } 1 \text { month) } \\
I_{\mathrm{b}} \text { (cumulative) } \\
\text { no effect } \\
N_{\mathrm{b}} \text { (constant } 1 \text { month) } \\
N_{\mathrm{b}} \text { (cumulative) } \\
I_{\mathrm{b}} \text { (constant } 1 \text { month) } \\
I_{\mathrm{b}} \text { (cumulative) } \\
\text { no effect }\end{array}$ & $\begin{array}{l}1633.2 \\
1707.0 \\
1567.9 \\
1627.4 \\
1562.1 \\
\mathbf{1 5 6 1 . 1} \\
1565.4 \\
1568.2 \\
1627.2 \\
1573.9\end{array}$ \\
\hline Plasmodium vivax $\times$ none & yes & $\begin{array}{l}N_{\mathrm{b}} \text { (constant } 1 \text { month) } \\
N_{\mathrm{b}} \text { (cumulative) } \\
I_{\mathrm{b}} \text { (constant } 1 \text { month) } \\
I_{\mathrm{b}} \text { (cumulative) } \\
\text { no effect } \\
N_{\mathrm{b}} \text { (constant } 1 \text { month) } \\
N_{\mathrm{b}} \text { (cumulative) } \\
I_{\mathrm{b}} \text { (constant } 1 \text { month) } \\
I_{\mathrm{b}} \text { (cumulative) } \\
\text { no effect }\end{array}$ & $\begin{array}{l}1623.4 \\
1717.0 \\
1563.4 \\
1620.5 \\
1574.3 \\
1565.7 \\
1562.2 \\
1564.2 \\
1620.3 \\
1562.2\end{array}$ \\
\hline
\end{tabular}

Notes: Species 1 indicates the focal parasite species, species 2 indicates whether a second parasite species was considered, "Inapparent infections" indicates whether the ratios for inapparent infections were considered, "Bed nets" indicates how the bed net effect was considered, and AIC refers to the Akaike information criterion value for the models (values in boldface type indicate the minimum for each species). Models are not directly comparable because they have different overdispersion parameters. NC indicates models whose parameters did not converge in the iterative process for fitting the negative binomial generalized linear models (NB-GLMs). When considering the second species, its $I^{\prime}$ was the best estimate for the species alone. $N_{\mathrm{b}}=N-$ (number of bed nets), where $N$ is the total host population; $I_{\mathrm{b}}=I^{\prime}$ - (number of bed nets), where $I^{\prime}$ is the number of parasitemic hosts based on previous and current cases.

TABLE 2. Parameter estimates for the best-fit models.

\begin{tabular}{lccccccc}
\hline \hline \multicolumn{1}{c}{ Species } & $\hat{\theta}$ & $\hat{\varphi}$ & $\hat{\beta}^{\prime}$ & $\hat{\beta}_{2}$ & $\hat{\alpha}$ & $\hat{k}$ & AIC \\
\hline P. falciparum & $0.737 \pm 0.050$ & $0.121 \pm 0.050$ & $0.842 \pm 0.343$ & $\dagger$ & $-0.106 \pm 0.028$ & $6.53 \pm 0.76$ & $1762.9(1766)$ \\
P. vivax & $0.648 \pm 0.058$ & $0.155 \pm 0.048$ & $0.820 \pm 0.240$ & $3.66 \pm 1.55$ & $-0.0796 \pm 0.0270$ & $7.58 \pm 0.96$ & $1561.1(1566.6)$
\end{tabular}

Notes: Parameter values (mean $\pm \mathrm{SE}$; defined in Methods) are assuming short times for parasite clearance (South Carolina patients). AIC values inside parentheses are for the same model assuming long times for parasite clearance (Georgia patients for malariotherapy).

$\uparrow$ Value not computed because of model structure. 

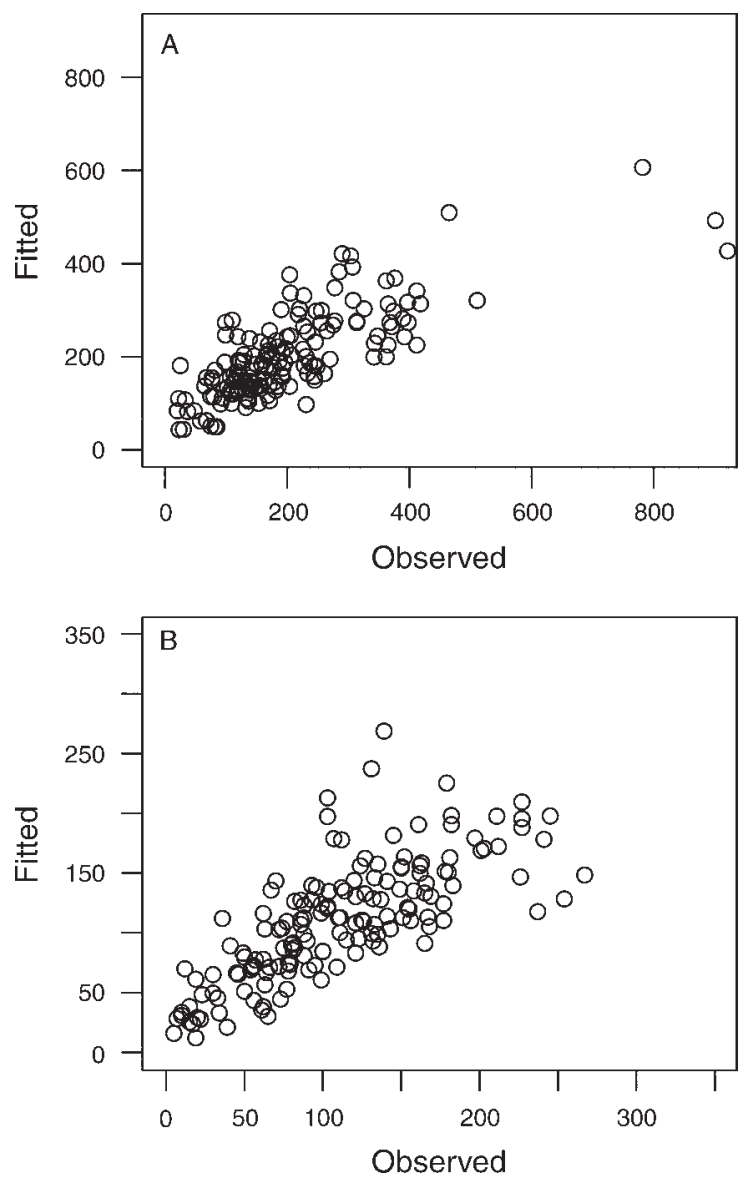

FIG. 4. Fitted vs. observed values for the best models. (A) Plasmodium falciparum malaria, Pearson's $r=0.77, \mathrm{CL}=[0.70$, 0.83]; (B) $P$. vivax malaria, Pearson's $r=0.74, \mathrm{CL}=[0.67,0.81]$.

fitted values is 0.77 for the best $P$. falciparum model, and 0.74 for the best model for $P$. vivax.

\section{Discussion}

Malaria models have been evolving (e.g., Bailey 1981, Anderson and May 1992, Awerbuch 1994) since the initial framework developed by Ross (1911). The results of our time-series model support the regulation of malaria infections by parasites present in the population. The process of model selection showed that the likelihood of models is minimized when a short-time of clearance ( 3 months) is used instead of a longer time (6 months). This suggests that lasting host immunity is not a major force regulating the population dynamics of infection. However, this result also indicates that either transient immunity to clear parasites is developed or the population-level use of anti-malarials has an effect in shortening clearance times (since the longer time estimate corresponds to immunologically naïve populations, Sama et al. 2006; see also Gurarie and McKenzie 2006, Klein et al. 2008).

The regulation of transmission by parasite abundance was originally proposed by Ross (1911) and it was the basis for future developments of control based on reducing mosquito populations. Strategies for mosquito control, primarily through mosquito larval habitat reduction (Kitron and Spielman 1989, Keiser et al. 2005, Spielman 2006) and bed nets (Kaneko et al. 2000, Lengeler 2004, Chaves et al. 2008), have been very successful, by contrast to vaccine trials (Druilhe and Barnwell 2007).

The transient effect of bed nets at the population level can be explained by the low local coverage associated with deliveries, which was about $5 \%$ of the population, when compared to the average of $80 \%$ seen at other localities (Kaneko et al. 2000, Chaves et al. 2008). Thus, the threshold for the population effectiveness of bed nets was never reached in this island. Although the effect of bed nets seems to be primarily one of reducing the total population at risk of infection, more sophisticated models and field studies are needed to understand their regulatory function.

The effect of climatic forcing in regulating transmission was small when compared to the endogenous factors regulating the population, and slightly larger for $P$. falciparum, in accordance with the effects seen for the whole archipelago of Vanuatu (Chaves et al. 2008). It is also worth to notice that in our specific study setting mosquitoes do not seem to be a limiting factor for transmission (Williams et al. 1995). However, for other settings transmission limitation by mosquito abundance may play a role and could amplify the effects of climate on transmission dynamics, thus calling for increased studies on mosquito ecology under changing environments.

Differences in seasonal patterns and age specific prevalence for $P$. vivax and $P$. falciparum have been used as evidence for heterologous (cross-specific) immunity for these two malaria parasites in Vanuatu (Maitland et al. 1996, 1997). However, the seasonal patterns are very similar for the two species over a longer time horizon (Fig. 2). Infection by one species does not seem to reduce the infection with the other, and in the biogeographical region where Vanuatu is located, multispecies infections are common, although only detected by sensitive molecular based techniques (Bruce et al. 2000, Bruce and Day 2003, Mayxay et al. 2004). However, the presence of co-infections at any given time does not necessarily indicate mechanisms of parasite species interactions.

Our results are consistent with observations from malariotherapy patients (Boyd and Kitchen 1937, 1938), where following a co-infection, $P$. falciparum reached higher densities first, in some cases suppressing the growth of $P$. vivax. They are also consistent with evidence on the absence of heterologous immunity (Collins and Jeffery 1999a, Molineaux et al. 2002). A lack of heterologous immunity may be explained by differences in resource exploitation by the two parasites inside the hosts, since $P$. falciparum is able to parasitize all erythrocytes whereas $P$. vivax only parasitizes young 
ones, leading to enhanced parasitemia by the former (McQueen and McKenzie 2006). This difference can also explain the dynamic sufficiency $P$. falciparum, since it can reach peak densities in the presence of $P$. vivax, whereas the latter reaches its maximum only after the former parasite is cleared (Boyd and Kitchen 1938).

For the interaction between the parasites to be positive at the population level, a mechanism of density-dependent within-host regulation is likely to be at play as proposed by Bruce and Day (2002). This is supported by empirical observations and theoretical results. The results by Bruce et al. (2000) suggest that parasites are likely to be regulated only when they reach large densities, since fluctuating densities of parasites through time were reported together with the development of tolerance to malaria parasites in malariotherapy patients (Molineaux et al. 2002). Also, levels of parasitemia in holoendemic settings are reported to become stabilized as individuals age (Ofulla et al. 2005) and significant reductions in malaria transmission have been reported after population-level drug administration campaigns that reduce within-host parasite density (e.g., Kaneko et al. 2000). Our results with loop analysis show that when the two parasites co-occur, a positive effect of a second parasite species on the first is plausible because of self-regulation of the former parasite as expected under a bottom-up mechanism (Fig. 1).

Another explanation for the dynamics observed here is a switch in immune strategy of individuals with age, since there is evidence for changes from nonspecific to specific immune responses in hyper-endemic settings (Rogier et al. 1999). More specifically, it has been shown that in early age infections, parasite clearance is mostly carried out by cells of the innate response, and that overexpression of Interferon gamma, (IFN- $\gamma$ ) and the tumor necrosis factor alpha (TNF- $\alpha$ ) facilitate phagocytosis of malaria parasites. By contrast, due to the lack of immune plasticity in adults, the immune response will depend mostly on $\mathrm{T}$ and $\mathrm{B}$ specific cells and their effector mechanisms (Artavanis-Tsakonas et al. 2003). The demographic profile of Vanuatu, including that of Espirito Santo, shows that at least $30 \%$ of the total population is under 15 , and most of the cases are concentrated in this age group (Kaneko et al. 1998, 2000). Although adults may be an important source of infections (Bousema et al. 2007), children are the main source of gametocytes (infecting stage to mosquitoes) in hyperendemic settings, where prevalence is above $20 \%$, as in Espirito Santo (Maitland et al. 1996). This would explain why long-lasting immune responses that can lead to cross-immunity do not play a major role in regulating the dynamics of transmission. Additionally, human traits like metabolic deficiencies on G6PD and $\alpha$-thalassemias could have dynamical effects similar to innate immunity (e.g., Kaneko et al. 2000).

Finally, our study emphasizes the need to better understand the factors regulating the dynamics of infection before formulating strategies of control at the population level. The failure of strategies that target infection management through immunity, e.g., vaccines, may ultimately be determined by their irrelevance to the regulation of disease dynamics at the population level.

\section{ACKNOWLEDGMENTS}

We thank the people of Vanuatu for their generosity over years of study. G. Taleo, K. Ichimori (at the World Health Organization), and T. Kurita compiled the data set. Valuable comments were provided by S. Allesina, A. Bhattarai, A. Björkman, N. Chitnis, I. Hwaihwanje, E. L. Ionides, C. Lengeler, N. Maire, B. Ngasala, D. J. Rodriguez, B. E. Sedio, T. Smith, J. H. Vandermeer, and two anonymous referees. Special thanks to M. L. Wilson, for guidance on loop analysis and suggestions for presenting this technique. L. F. Chaves was funded by Fundación Polar, Caracas, Venezuela, the University of Michigan through the Graham Environmental Sustainability Institute (to M. Pascual), the Rackham Graduate School Research Grant, the Department of Ecology and Evolutionary Biology Block Grant and Summer Fellowship, the International Institute, and the Program in Global Health, School of Public Health (to M. L. Wilson). A. Kaneko was funded by the Swedish Foundation for International Cooperation in Research and Higher Education (STINT), the Swedish Research Council (VR, 2005-6836) and Global COE Program, and Japan's Ministry of Education, Culture, Sports, Science, and Technology (MEXT), 2008-2013. M. Pascual was supported by the National Oceanic and Atmospheric Administration (Oceans and Health Program NA 040 AR 460019) and NSF-NIH (Ecology of Infectious Diseases Grant EF 0430 120). M. Pascual is also an investigator of the Howard Hughes Medical Institute.

\section{Literature Cited}

Anderson, R. M., and R. M. May. 1992. Infectious diseases of humans. Oxford University Press, Oxford, UK.

Artavanis-Tsakonas, K., J. E. Tongren, and E. M. Riley. 2003. The war between the malaria parasite and the immune system: immunity, immunoregulation and immunopathology. Clinical and Experimental Immunology 133:145-152.

Awerbuch, T. 1994. Evolution of mathematical models of epidemics. Annals of the New York Academy of Sciences 740:232-241.

Bailey, N. T. J. 1981. The biomathematics of malaria. Griffin, London, UK.

Berryman, A. A., M. Lima, and B. A. Hawkins. 2002. Population regulation, emergent properties, and a requiem for density dependence. Oikos 99:600-606.

Bousema, J. T., C. J. Drakeley, J. Kihonda, J. C. M. Hendriks, N. I. J. Akim, W. Roeffen, and R. W. Sauerwein. 2007. A longitudinal study of immune responses to Plasmodium falciparum sexual stage antigens in Tanzanian adults. Parasite Immunology 29:309-317.

Boyd, M. F., and S. F. Kitchen. 1937. Simultaneous inoculation with Plasmodium vivax and Plasmodium falciparum. American Journal of Tropical Medicine and Hygiene 17:855-861.

Boyd, M. F., and S. F. Kitchen. 1938. Vernal vivax activity in persons simultaneously inoculated with Plasmodium vivax and Plasmodium falciparum. American Journal of Tropical Medicine and Hygiene 18:505-514.

Bruce, M. C., and K. P. Day. 2002. Cross-species regulation of malaria parasitaemia in the human host. Current Opinion in Microbiology 5:431-437.

Bruce, M. C., C. A. Donnelly, M. P. Alpers, M. R. Galinski, J. W. Barnwell, D. Walliker, and K. P. Day. 2000. Crossspecies interactions between malaria parasites in humans. Science 287:845-848.

Cannon, D. S. 1958. Malaria and prematurity in the western region of Nigeria. British Medical Journal 2:877-878. 
Cazelles, B., and N. P. Chau. 1997. Using the Kalman filter and dynamic models to assess the changing HIV/AIDS epidemic. Mathematical Biosciences 140:131-154.

Chaves, L. F. 2007. Casas muertas and Oficina No. 1: internal migrations and malaria trends in Venezuela 1905-1945. Parasitology Research 101:19-23.

Chaves, L. F., A. Kaneko, G. Taleo, M. Pascual, and M. L. Wilson. 2008. Malaria transmission pattern resilience to climatic variability is mediated by insecticide treated nets. Malaria Journal 7:100.

Chaves, L. F., and M. Pascual. 2006. Climate cycles and forecasts of cutaneous leishmaniasis, a nonstationary vectorborne disease. PLoS Medicine 3:1320-1328.

Cohen, J. E. 1973. Heterologous immunity in human malaria. Quarterly Review of Biology 48:467-489.

Cohen, J. E. 1995. Population growth and earth's human carrying capacity. Science 269:341-346.

Collins, W. E., and G. M. Jeffery. 1999a. A retrospective examination of secondary sporozoite- and trophozoiteinduced infections with Plasmodium falciparum: development of parasitologic and clinical immunity following secondary infection. American Journal of Tropical Medicine and Hygiene 61:20-35.

Collins, W. E., and G. M. Jeffery. 1999b. A retrospective examination of sporozoite- and trophozoite-induced infections with Plasmodium falciparum: development of parasitologic and clinical immunity during primary infection. American Journal of Tropical Medicine and Hygiene 61:419.

de Jong, M. C. M. 1995. How does transmission of infection depend on population size? Pages 84-94 in D. Mollison, editor. Epidemic models, their structure and relation to data. Cambridge University Press, Cambridge, UK.

Dietz, K., L. Molineaux, and A. Thomas. 1974. Malaria model tested in African savannah. Bulletin of the World Health Organization 50:347-357.

Druilhe, P., and J. W. Barnwell. 2007. Pre-erythrocytic stage malaria vaccines: time for a change in path. Current Opinion in Microbiology 10:371-378.

Durbin, J., and S. J. Koopman. 2001. Time series analysis by state space methods. Oxford University Press, Oxford, UK.

Ellner, S. P., Y. Seifu, and R. H. Smith. 2002. Fitting population dynamic models to time-series data by gradient matching. Ecology 83:2256-2270.

Färnert, A., G. Snounou, I. Rooth, and A. Björkman. 1997. Daily dynamics of Plasmodium falciparum subpopulations in asymptomatic children in a holoendemic area. American Journal of Tropical Medicine and Hygiene 56:538-547.

Felger, I., T. Smith, D. Edoh, A. Kitua, P. Alonso, M. Tanner, and H. P. Beck. 1999. Epidemiology of multiple Plasmodium falciparum infections. 6. Multiple Plasmodium falciparum infections in Tanzanian infants. Transactions of the Royal Society of Tropical Medicine and Hygiene 93:S29-S34.

Fenton, A., J. P. Fairbairn, R. Norman, and P. J. Hudson. 2002. Parasite transmission: reconciling theory and reality. Journal of Animal Ecology 71:893-905.

Filipe, J. A., E. M. Riley, C. J. Drakeley, C. J. Sutherland, and A. C. Ghani. 2007. Determination of the processes driving the acquisition of immunity to malaria using a mathematical transmission model. PLoS Computational Biology 3:e255.

Finkenstädt, B. F., and B. T. Grenfell. 2000. Time series modelling of childhood diseases: a dynamical systems approach. Journal of the Royal Statistical Society Series C 49:187-205.

Gause, G. F. 1934. The struggle for existence. Williams and Wilkins, Baltimore, Maryland, USA.

Gill, C. A. 1928. The genesis of epidemics and the natural history of disease. An introduction to the science of epidemiology based upon the study of epidemics of malaria, influenza and plague. Bailliere, Tindall, and Cox, London, UK.
Gill, C. A. 1938. The seasonal periodicity of malaria and the mechanism of the epidemic wave. J. and A. Churchill Ltd., London, UK.

Gupta, S., and K. P. Day. 1994a. A strain theory of malaria transmission. Parasitology Today 10:476-481.

Gupta, S., and K. P. Day. 1994b. A theoretical framework for the immunoepidemiology of Plasmodium falciparum malaria. Parasite Immunology 16:361-370.

Gurarie, D., and F. E. McKenzie. 2006. Dynamics of immune response and drug resistance in malaria infection. Malaria Journal 5:86.

Gurarie, D., P. A. Zimmerman, and C. H. King. 2006. Dynamic regulation of single- and mixed-species malaria infection: insights to specific and non-specific mechanisms of control. Journal of Theoretical Biology 240:185-199.

Hochberg, M. E. 1991. Nonlinear transmission rates and the dynamics of infectious disease. Journal of Theoretical Biology 153:301-321.

Kaneko, A., G. Taleo, M. Kalkoa, S. Yamar, T. Kobayakawa, and A. Björkman. 2000. Malaria eradication on islands. Lancet 356:1560-1564.

Kaneko, A., G. Taleo, M. Kalkoa, J. Yaviong, P. A. Reeve, M. Ganczakowski, C. Shirakawa, K. Palmer, T. Kobayakawa, and A. Björkman. 1998. Malaria epidemiology, glucose 6phosphate dehydrogenase deficiency and human settlement in the Vanuatu Archipelago. Acta Tropica 70:285-302.

Keiser, J., B. H. Singer, and J. Utzinger. 2005. Reducing the burden of malaria in different eco-epidemiological settings with environmental management: a systematic review. Lancet Infectious Diseases 5:695-708.

Kitron, U., and A. Spielman. 1989. Suppression of transmission of malaria through source reduction: antianopheline measures applied in Israel, the United States, and Italy. Reviews of Infectious Diseases 11:391-406.

Klein, E., D. Smith, M. Boni, and R. Laxminarayan. 2008. Clinically immune hosts as a refuge for drug-sensitive malaria parasites. Malaria Journal 7:67.

Kot, M., W. M. Schaffer, G. L. Truty, D. J. Graser, and L. F. Olsen. 1988. Changing criteria for imposing order. Ecological Modelling 43:75-110.

Kuby, J. 1997. Immunology. W. H. Freeman, New York, New York, USA.

Lengeler, C. 2004. Insecticide-treated bed nets and curtains for preventing malaria. Cochrane Database of Systematic Reviews:CD000363.

Levins, R. 1968. Evolution in changing environments. Princeton University Press, Princeton, New Jersey, USA.

Levins, R. 1969. Effect of random variations of different types on population growth. Proceedings of the National Academy of Sciences (USA) 62:1061-1065.

Levins, R. 1974. The qualitative analysis of partially specified systems. Annals of the New York Academy of Sciences 231: $123-138$

Lewontin, R. C. 1974. The genetic basis of evolutionary change. Columbia University Press, New York, New York, USA.

Lewontin, R. C., and D. Cohen. 1969. On population growth in a randomly varying environment. Proceedings of the National Academy of Sciences (USA) 62:1056-1060.

Liu, W. M., H. W. Hethcote, and S. A. Levin. 1987. Dynamic behavior of epidemiologic models with nonlinear incidence rates. Journal of Mathematical Biology 25:359-380.

Maitland, K., T. N. Williams, S. Bennett, C. I. Newbold, T. E. A. Peto, J. Viji, R. Timothy, J. B. Clegg, D. J. Weatherall, and D. K. Bowden. 1996. The interaction between Plasmodium falciparum and P. vivax in children on Espiritu Santo island, Vanuatu. Transactions of the Royal Society of Tropical Medicine and Hygiene 90:614-620.

Maitland, K., T. N. Williams, and C. I. Newbold. 1997. Plasmodium vivax and P. falciparum: Biological interactions and the possibility of cross-species immunity. Parasitology Today 13:227-231. 
Mardsen, P. D. 1964. The Sukuta project. A longitudinal study of health in Gambian children from birth to 18 months age. Transactions of the Royal Society of Tropical Medicine and Hygiene 58:455-489.

Matzinger, P. 1994. Tolerance, danger, and the extended family. Annual Review of Immunology 12:991-1045.

Mayxay, M., S. Pukrittayakamee, P. N. Newton, and N. J. White. 2004. Mixed-species malaria infections in humans. Trends in Parasitology 20:233-240.

McKenzie, F. E., and W. H. Bossert. 1997. Mixed-species Plasmodium infections of humans. Journal of Parasitology 83:593-600.

McKenzie, F. E., and W. H. Bossert. 2005. An integrated model of Plasmodium falciparum dynamics. Journal of Theoretical Biology 232:411-426.

McQueen, P. G., and F. E. McKenzie. 2006. Competition for red blood cells can enhance Plasmodium vivax parasitemia in mixed-species malaria infections. American Journal of Tropical Medicine and Hygiene 75:112-125.

Mehlotra, R. K., K. Lorry, W. Kastens, S. M. Miller, M. P. Alpers, M. Bockarie, J. W. Kazura, and P. A. Zimmerman. 2000. Random distribution of mixed species malaria infections in Papua New Guinea. American Journal of Tropical Medicine and Hygiene 62:225-231.

Mitchell, T. D., M. Hulme, and M. New. 2002. Climate data for political areas. Area 34:109-112.

Molineaux, L., and G. Gramiccia. 1980. The Garki Project: research on the epidemiology and control in the Sudan savanna of West Africa. World Health Organization, Geneva, Switzerland.

Molineaux, L., J. Storey, J. E. Cohen, and A. Thomas. 1980. A longitudinal-study of human malaria in the west-African savanna in the absence of control measures - relationships between different plasmodium species, in particular $P$. falciparum and Plasmodium-malariae. American Journal of Tropical Medicine and Hygiene 29:725-737.

Molineaux, L., M. Trauble, W. E. Collins, G. M. Jeffery, and K. Dietz. 2002. Malaria therapy reinoculation data suggest individual variation of an innate immune response and independent acquisition of antiparasitic and antitoxic immunities. Transactions of the Royal Society of Tropical Medicine and Hygiene 96:205-209.

Nedelman, J. 1984. Inoculation and recovery rates in the malaria model of Dietz, Molineaux, and Thomas. Mathematical Biosciences 69:209-233.

Nedelman, J. 1985. Some new thoughts about some old malaria models: introductory review. Mathematical Biosciences 73: $159-182$.

Nelder, J. A., and R. Mead. 1965. A simplex-method for function minimization. Computer Journal 7:308-313.

Ofulla, A. V., A. M. Moormann, P. E. Embury, J. W. Kazura, P. O. Sumba, and C. C. John. 2005. Age-related differences in the detection of Plasmodium falciparum infection by PCR and microscopy, in an area of Kenya with holo-endemic malaria. Annals of Tropical Medicine and Parasitology 99: 431-435.

Pascual, M., B. Cazelles, M. J. Bouma, L. F. Chaves, and K. Koelle. 2008. Shifting patterns: malaria dynamics and rainfall variability in an African highland. Proceedings of the Royal Society B 275:123-132.

Priestley, M. B. 1988. Non-linear and non-stationary time series analysis. Academic Press, London, UK.
Puccia, C. J., and R. Levins. 1985. Qualitative modeling of complex systems. Harvard University Press, Cambridge, Massachusetts, USA.

Richie, T. L. 1988. Interactions between malaria parasites infecting the same vertebrate host. Parasitology 96:607-639.

Rogier, C., A. B. Ly, A. Tall, B. Cisse, and J. F. Trape. 1999. Plasmodium falciparum clinical malaria in Dielmo, a holoendemic area in Senegal: no influence of acquired immunity on initial symptomatology and severity of malaria attacks. American Journal of Tropical Medicine and Hygiene 60: 410-420.

Ross, R. 1911. The prevention of Malaria. John Murray, London, USA.

Roy, M., and M. Pascual. 2006. On representing network heterogeneities in the incidence rate of simple epidemic models. Ecological Complexity 3:80-90.

Royama, T. 1992. Analytical population dynamics. Chapman and Hall, London, UK.

Sama, W., K. Dietz, and T. Smith. 2006. Distribution of survival times of deliberate Plasmodium falciparum infections in tertiary syphilis patients. Transactions of the Royal Society of Tropical Medicine and Hygiene 100:811-816.

Schmalhausen, I. I. 1949. Factors of evolution; the theory of stabilizing selection. Blakiston Company, Philadelphia, Pennsylvania, USA.

Shumway, R. H., and D. S. Stoffer. 2000. Time series analysis and its applications. Springer, New York, New York, USA.

Sinclair, A. R. E. 1989. The regulation of animal populations. Pages 197-241 in J. M. Cherrett, editor. Ecological concepts. Blackwell, Oxford, UK.

Smith, T., H. P. Beck, A. Kitua, S. Mwankusye, I. Felger, N. Fraser-Hurt, A. Irion, P. Alonso, T. Teuscher, and M. Tanner. 1999. Epidemiology of multiple Plasmodium falciparum infections. 4. Age dependence of the multiplicity of Plasmodium falciparum infections and of other malariological indices in an area of high endemicity. Transactions of the Royal Society of Tropical Medicine and Hygiene 93:S15-S20.

Smith, T., B. Genton, K. Baea, N. Gibson, A. Narara, and M. P. Alpers. 2001. Prospective risk of morbidity in relation to malaria infection in an area of high endemicity of multiple species of Plasmodium. American Journal of Tropical Medicine and Hygiene 64:262-267.

Spielman, A. 2006. Ethical dilemmas in malaria control. Journal of Vector Ecology 31:1-8.

Struchiner, C. J., M. E. Halloran, and A. Spielman. 1989. Modeling malaria vaccines. 1. New uses for old ideas. Mathematical Biosciences 94:87-113.

Turchin, P. 2003. Complex population dynamics. Princeton University Press, Princeton, New Jersey, USA.

Vandermeer, J. H. 1969. Competitive structure of communities: an experimental approach with protozoa. Ecology 50:362371.

Williams, T. N., K. Maitland, and D. H. Foley. 1995. Malaria sporozoite rates for Anopheles farauti s.s. Laveran (Diptera, Culicidae) from Vanuatu. Annals of Tropical Medicine and Parasitology 89:305-307.

Zavaleta, J. O., and P. A. Rossignol. 2004. Community level analysis of risk of vector-borne disease. Transactions of the Royal Society of Tropical Medicine and Hygiene 98:610618 . 